\title{
The mental health status of ethnocultural minorities in Ontario and their mental health care
}

\author{
Sherry L. Grace ${ }^{1,2,5^{*}}$, Yongyao Tan ${ }^{2}$, Robert A. Cribbie ${ }^{3}$, Han Nguyen ${ }^{4}$, Paul Ritvo ${ }^{1}$ and Jane Irvine ${ }^{3}$
}

\begin{abstract}
Background: Mental disorders are a leading cause of disability and early mortality. The objective of this study was to describe and compare psychosocial indicators and mental health service use among ethnoculturallydiverse Ontarians.

Methods: This is a cross-sectional analysis of the Ontario Health Study pilot investigation. Residents were mailed an invitation to one of 3 assessment centres (urban, rural and northern sites) from March 2009 to July 2010. Participants had an interview with a nurse and completed a questionnaire on a touchscreen kiosk. The questionnaire included sociodemographic items, and scales assessing symptoms of depressive symptoms (CES-D) and anxiety (GAD-7), social support (Lubben Social Network Scale), stressful life events, and mental health service use.

Results: Eight thousand two hundred thirty-five residents participated, among whom 6652 (82.4 \%) self-reported their ethnocultural background as White, 225 (2.8 \%) as South Asian, 222 (2.8 \%) East Asian, 214 (2.7 \%) Southeast Asian, 197 (2.4\%) Black, and 28 (0.3\%) as Aboriginal. Based on their sociodemographic characteristics, participants from these ethnocultural minority groups were matched to White participants. Black participants reported significantly greater stressful life events than White participants $(p=.04)$, particularly death $(p<.05)$, divorce $(p=.002)$ and financial difficulties $(p<.001)$. East Asian participants reported significantly less social support than their White counterparts $(p<.001)$, and this was not confounded by measurement variance. Mental health service use was significantly lower in all ethnocultural minorities except Aboriginals, when compared to White participants $(p=.001)$.
\end{abstract}

Conclusions: There is a high burden of psychosocial distress in several preponderant ethnocultural minorities in Ontario; many of whom are not accessing available mental health services.

Keywords: Mental health, Ethnic groups, Emigrants and immigrants, Ontario

\section{Background}

As the second leading cause of human disability and premature death, mental disorders are a significant public health concern [1]. Current estimates suggest one in five Canadians will experience a mental illness in their lifetime $[2,3]$. In addition, mental health problems account for $\$ 51$ billion in annual spending [2], making it one of the costliest healthcare burdens in Canada [4].

\footnotetext{
*Correspondence: sgrace@yorku.ca

${ }^{1}$ School of Kinesiology and Health Science, York University, Toronto, ON, Canada

${ }^{2}$ Cardiac Rehabilitation and Prevention Program, Toronto Rehabilitation Institute, University Health Network, Toronto, ON, Canada

Full list of author information is available at the end of the article
}

Anxiety disorders and depressive symptoms are two of the most prevalent mental disorders. Both anxiety and depressive symptoms can take a chronic debilitating course, with depressive symptoms particularly related to increased morbidity and mortality from medical conditions and decreased quality of life, among other consequences $[5,6]$. Beyond mental disorders, social support and stressful life events have also been shown to affect an individual's mental health and psychosocial wellbeing [7]. Individuals need not suffer with mental illness in Canada without treatment, as evidence-based treatments for the most common mental disorders [8], can be accessed within the provincial healthcare systems. 
The term "ethnocultural minority" describes people of a "culturally and linguistically-diverse background", where culture consists of a system of values, norms, and beliefs that shape an individual's daily experiences and behavior [9]. In Canada, the most populous ethnocultural groups are South Asian (e.g., India, Pakistan, Bangladesh, and Sri Lanka; $4.8 \%$ ), Aboriginal (4.3\%), Chinese (4.0\%), and Black (2.9\%). As per the 2011 National Household Survey, 6,775,800 individuals were foreign-born, representing $20.6 \%$ of the total population [10], and approximately 250,000 people immigrate to Canada each year [11]. Individuals from South Asia are the largest and fastest growing group.

The literature regarding mental health in Canada illustrates that despite the country's diversity, there are very few comprehensive research studies regarding psychosocial well-being or access to mental health services among ethnocultural groups [12]. Ethnocultural minorities experience greater exposure to the negative effects of some social determinants of health, such as income insecurity and social isolation [12]. Immigrants and refugees are separated from their social supports, which makes them at the increased risk of mental health problems and illnesses [13]. Visible minorities often face racial discrimination. Finally, Aboriginal peoples in Canada are known to suffer poorer mental health, and an alarmingly high rate of suicide is observed [14].

Stakeholders have called for better research into these populations in Canada, arguing that more in-depth investigation may uncover the key processes that give rise to mental health risk [15]. Accordingly, the objective of this study was to describe and compare depressive and anxiety symptoms, stressful life events, perceived social support and mental health service use among ethnoculturally-diverse Ontarians.

\section{Methods}

\section{Design and procedure}

This cross-sectional study was based on data from the Ontario Health Study (OHS) pilot. The OHS aims to investigate how lifestyle, environment and family history factors increase the risk of common chronic diseases such as cancer, diabetes, and heart disease [16]. Data were collected at three assessment centres from March 2009 to July 2010. Potential participants were invited to attend an assessment centre to participate via mail. At the centres, participants completed a touchscreen questionnaire which included psychosocial assessments. Participants also had an interview with a nurse to provide personal and family medical history and prescription medications. A data access application for this study was approved by the OHS. Ethics approval was secured through the York University and University of Toronto Research Ethics Boards.

\section{Participants}

Participants were recruited from the communities of Mississauga, Oakville, Brampton, Burlington, Owen Sound, and Sudbury, Ontario, Canada. These communities were selected to achieve representation of rural (Owen Sound), northern (Sudbury), and urban (all others) Ontarians in the sample. The urban centres are in the Greater Toronto Area, where a large proportion of Canadian immigrants settle [10]. Potential participants $(N=306,041)$ were identified from purchased mailing lists. Eligible participants had to be residents of Ontario, between the ages of 35-69 years, and sufficiently proficient in English or French. Participants provided written informed consent for participation in the study.

\section{Measures}

Sociodemographic characteristics and mental health were assessed in the self-report survey. Participants were asked to report their ethnocultural background from the following options: Aboriginal (e.g., First Nations, Métis or Inuit), Arab (e.g., Egypt, Iraq, Jordan, Lebanon), Black (African or Caribbean descent), East Asians (i.e., China, Japan, Korea, Taiwan), Filipino, Jewish, Latin American/ Hispanic, South Asian (e.g., India, Sri Lanka, Pakistan, Bangladesh), Southeast Asian (e.g., Malaysia, Indonesia, Vietnam), West Asian (e.g., Turkey, Iran, Afghanistan), White and other. Finally, participants were also asked to rate their health status, on a scale from $1=$ poor, to $5=$ excellent.

\section{Psychosocial Well-Being}

Depressive and anxiety symptoms, social support, and stressful life events were assessed. Depressive symptoms were measured using the Center for Epidemiological Studies Depressive symptoms Scale (CES-D) [17]. It is a 20-item self-report scale designed to measure depressive symptomatology in the general population. Scores range from 0 to 60 , where higher scores indicate more symptoms, weighted by frequency of occurrence during the past week. The threshold for elevated depressive symptoms on the CES-D-20 is often interpreted as a score of 16 or greater. The scale has been validated in African American, Asian American, Asian, Chinese, French, Greek, Hispanic, Japanese, Serbian, and East Indian samples $[18,19]$. Good reliability, excellent internal consistency (coefficient alpha $>0.85$ in community samples and 0.9 in psychiatric samples), and good testretest correlation $(r=.65)$ have been demonstrated. The CES-D also has high sensitivity and specificity [20].

Anxiety symptoms were measured using the Generalized Anxiety Disorder scale (GAD-7) [21]. It consists of seven items, each of which is scored 0 to 3 , providing a total severity score ranging from 0 to 21 . Cutpoints of 5 , 
10 , and 15 represent mild, moderate, and severe levels of anxiety on the scale. Although originally developed to assess generalized anxiety disorder, the GAD-7 has also proved to have good sensitivity and specificity as a screener for panic, social anxiety, and post-traumatic stress disorder [22].

The Lubben Social Network Scale (LSNS-6) [23] is designed to gauge perceived social support received by family and friends. Scores range from 0 to 30, with higher scores indicating a greater level of social support and low risk for isolation. Scores less than 6 on the family or friend subscales suggest marginal ties, with overall scores less than 12 indicative of social isolation. The 6-item version has been demonstrated to be reliable (Cronbach's $\alpha=0.83$ ), and to discriminate between socially-connected versus isolated participants [23].

The Total Life Events Scale measures stressful life events in the past year. The scale administered was a slightly modified version of the scale administered by the United Kingdom Biobank [24] and INTERHEART [25]. It is a 12-item questionnaire where respondents are asked whether they have experienced a specific event. Scores range from 0 to 12, with higher scores indicating a greater impact of stressful life events.

Finally, participants were asked to report whether they had visited a mental healthcare provider for "nerves, anxiety, tension or depressive symptoms" and to report which type of provider. This item was investigatorgenerated. Participants could check all the response alternatives that were applicable.

\section{Statistical analyses}

IBM SPSS Statistics Software Version 21 was used for preliminary statistical analyses. Ethnocultural backgrounds for which there were more than 150 participants were selected for further analysis. This size was chosen so that upon propensity-matching, a sufficient sample would be available for robust comparison to White participants. However, Aboriginal participants were also selected for analysis regardless of size, given the known mental health disparities between Aboriginals and the general population [14]. A comparison of participant sociodemographic characteristics by ethnocultural background was performed.

In order to compare the White participants to the most common ethnocultural minority groups and Aboriginal participants, we matched the participants on important sociodemographic variables known to be related to mental health. Using the variables of sex, education, house ownership and income, participants were matched using the $\mathrm{R}$ package MatchIt [26]. Given the categorical nature of the demographic variables, exact matching was utilized.
To test for ethnocultural differences in psychosocial indicators, for the categorical outcome variables, Fisher's exact test was used to compare frequencies across groups. This was undertaken for both the non-matched and matched samples, to enable a more fulsome understanding of the robustness of any associations observed. For continuous outcome variables the heteroscedastic Welch $t$ test was used to compare the ethnocultural groups (non-matched and matched). For the variables depressive symptoms, anxiety, stressful life events and total mental health services use, the square root of the variables was used, since this brought the distribution of the variables towards normality.

In order to investigate the role that measurement invariance might play in psychosocial differences between the ethnocultural groups, if the matched groups differed on the well-validated scales, namely the CES-D, GAD-7 or Lubben scales, a multiple group structural equation model was used to determine if any of the loadings or factor covariances differed [27]. Item and factor intercepts/means were not assessed for invariance since those relate to the main hypotheses of the study. Model fit was assessed using the model chi-square statistic, root mean square error of approximation (RMSEA) and comparative fit index (CFI). For the RMSEA, values less than .06 are indicative of a good fitting model, and for the CFI values greater than .95 are indicative of a good fitting model (Hu \& Bentler, 1999). These analyses were undertaken using $\mathrm{R}$ version 3.1.0 [28].

\section{Results}

\section{Respondent characteristics}

The sample consisted of 8235 participants (2.69 \% response rate). Of the 259,159 urban Ontarians mailed, $5,228(2.02 \%)$ participated; of the 13,456 rural Ontarians mailed, 1,366 (10.2\%) participated; and of the 33,417 northern Ontarians mail, 1,641 (4.91 \%) participated.

Table 1 describes self-reported ethnocultural background of participants. As shown, the most frequent ethnocultural backgrounds were White, South Asian, East Asian, Southeast Asian, and Black. This is somewhat consistent with the distribution of visible minorities in Ontario, namely South Asian (26\%), East Asian (22 \%), Black (19\%), Filipino (7 \%), Latin American (5\%), Arab (4\%), Southeast Asian (4\%), West Asian (3\%), and Korean (3\%), among others (http://www.fin.gov.on.ca/en/ economy/demographics/census/cenhi6e.pdf). The proportion of Aboriginal respondents was $0.3 \%$, compared to $2.4 \%$ in Ontario broadly [29].

Participants' sociodemographic characteristics are shown in Table 2. Overall, participants were a mean of $55.64 \pm 8.89$ years old (standard deviation). Also shown in Table 2 is a comparison of participants' sociodemographic characteristics by ethnocultural background. 
Table 1 Self-reported ethnocultural background of OHS pilot study respondents

\begin{tabular}{lll}
\hline & $n$ & $\%$ \\
\hline White & 6652 & 82.4 \\
South Asian & 225 & 2.8 \\
East Asian & 222 & 2.8 \\
Southeast Asian & 214 & 2.7 \\
Black & 197 & 2.4 \\
Latin American/Hispanic & 102 & 1.3 \\
Filipino & 66 & 0.8 \\
Arab & 43 & 0.5 \\
Jewish & 32 & 0.4 \\
Aboriginal & 28 & 0.3 \\
West Asian & 20 & 0.2 \\
Multiple Ethnicities & 193 & 2.2 \\
Other & 86 & 1.1 \\
\hline
\end{tabular}

Compared to White participants, there were significantly fewer female South Asian, East Asian and Southeast Asian participants, and significantly more female Aboriginal participants. With regard to highest educational attainment, East Asian and Southeast Asian participants reported significantly higher education than White participants. With regard to first language spoken, significantly more White participants reported English as their first language than South Asian, East Asian and Southeast Asian. However, Black participants were significantly more likely to report English as their first language than White participants. With regard to marital status, significantly more South Asian, East Asian and Southeast Asian participants reported being married or living with a partner than White participants. However, Black participants were significantly less likely than White participants to report being married or living with a partner. With regard to work status, South Asian, East Asian, Southeast Asian, and Black participants were significantly more likely to be working full-time than their White counterparts. With regard to household income, significantly more Black and Aboriginal participants reported earning less than $\$ 100,000$ CAD annually than White participants. With regard to home ownership status, significantly fewer Black participants reported owning a home than White participants. With regard to health status, significantly fewer South Asian, East Asian, Southeast Asian and Black participants reported their health in general is Good or better than White participants.

Of the complete cases, 100 (96.2\%) of the 104 Black, 111 (98.2 \%) of the 113 South Asian, 132 (98.5\%) of the 134 East Asian, 106 (95.5\%) of the 111 Southeast Asian, and 20 (95.2\%) of the 21Aboriginal participants had matches with the White participants. Of the 5120 White participants, 2083 (40.7\%) matched the characteristics of the included Black participants, 2115 (41.3\%) matched the characteristics of the included South Asian participants, 2486 (48.6 \%) matched the characteristics of the included East Asian participants, 1898 (37.1\%) matched the characteristics of the included Southeast Asian participants, and 518

Table 2 Characteristics of participants, by ethnocultural background

\begin{tabular}{|c|c|c|c|c|c|c|c|}
\hline & \multicolumn{7}{|l|}{ n (\%) } \\
\hline & $\begin{array}{l}\text { White } 6652 \\
(82.4)\end{array}$ & $\begin{array}{l}\text { South Asian } \\
225(2.8)\end{array}$ & $\begin{array}{l}\text { East Asian } \\
222(2.8)\end{array}$ & $\begin{array}{l}\text { Southeast Asian } \\
214(2.7)\end{array}$ & $\begin{array}{l}\text { Black } \\
197(2.4)\end{array}$ & $\begin{array}{l}\text { Aboriginal } \\
28(0.3)\end{array}$ & $\begin{array}{l}\text { Total } \\
N=7538(100)\end{array}$ \\
\hline Sex (Female) & $3716(55.9)$ & $\begin{array}{l}81(36.0) \\
(p<0.001)\end{array}$ & $\begin{array}{l}94(42.3) \\
(p<0.001)\end{array}$ & $\begin{array}{l}68(31.8) \\
(p<0.001)\end{array}$ & $122(61.9)$ & $\begin{array}{l}21(75.0) \\
(p<0.001)\end{array}$ & $4102(54.4)$ \\
\hline $\begin{array}{l}\text { Education } \\
\text { (Greater than high school) }\end{array}$ & $5182(78.2)$ & $186(83.0)$ & $\begin{array}{l}199(89.6) \\
(p<0.001)\end{array}$ & $\begin{array}{l}193(90.6) \\
(p<0.001)\end{array}$ & $155(78.7)$ & $18(64.3)$ & $5933(79.0)$ \\
\hline First Language (English) & $5493(82.6)$ & $\begin{array}{l}95(42.4) \\
(p<0.001)\end{array}$ & $\begin{array}{l}49(22.3) \\
(p<0.001)\end{array}$ & $\begin{array}{l}94(43.9) \\
(p<0.001)\end{array}$ & $\begin{array}{l}185(94.4) \\
(p<0.001)\end{array}$ & $21(75.0)$ & $5937(78.8)$ \\
\hline Marital Status (Married or living with a partner) & $5363(80.7)$ & $\begin{array}{l}206(92.0) \\
(p<0.001)\end{array}$ & $\begin{array}{l}199(89.6) \\
(p<0.001)\end{array}$ & $\begin{array}{l}198(92.5) \\
(p<0.001)\end{array}$ & $\begin{array}{l}122(62.2) \\
(p<0.001)\end{array}$ & $25(89.3)$ & $6113(81.2)$ \\
\hline Work Status (Full-time) & $3069(46.1)$ & $\begin{array}{l}150(66.7) \\
(p<0.001)\end{array}$ & $\begin{array}{l}136(61.3) \\
(p<0.001)\end{array}$ & $\begin{array}{l}141(65.9) \\
(p<0.001)\end{array}$ & $\begin{array}{l}124(62.9) \\
(p<0.001)\end{array}$ & $14(50.0)$ & $3634(48.2)$ \\
\hline $\begin{array}{l}\text { Household Income } \\
(<\$ 100,000 \text { CAD })\end{array}$ & $3497(52.6)$ & $122(54.2)$ & $112(50.5)$ & $127(59.3)$ & $\begin{array}{l}121(61.4) \\
(p=0.008)\end{array}$ & $\begin{array}{l}21(75.0) \\
(p=0.008)\end{array}$ & $4000(53.1)$ \\
\hline Own Home (yes) & $6260(94.2)$ & $207(92.4)$ & $213(96.4)$ & $201(94.4)$ & $\begin{array}{l}175(89.3) \\
(p=0.043)\end{array}$ & $24(85.7)$ & $7080(94.1)$ \\
\hline $\begin{array}{l}\text { Perceived Health } \\
\text { (Good to Excellent) }\end{array}$ & $6332(95.3)$ & $\begin{array}{l}195(86.7) \\
(p<0.001)\end{array}$ & $\begin{array}{l}198(89.6) \\
(p<0.001)\end{array}$ & $\begin{array}{l}191(89.3) \\
(p<0.001)\end{array}$ & $\begin{array}{l}175(89.7) \\
(p<0.001)\end{array}$ & $25(89.3)$ & $7116(94.5)$ \\
\hline
\end{tabular}

Note: $p$ values show comparison between Non-White and White participants 
(10.1\%) matched the characteristics of the included Aboriginal participants.

\section{Psychosocial well-being}

In the overall sample, 3471 (46.4 \%) participants reported elevated depressive symptoms (i.e., CES-D scores greater than 16). With regard to anxiety, $1022(13.6 \%)$ participants reported mild, 324 (4.3\%) participants reported moderate, and $153(2.0 \%)$ participants reported severe symptoms. With regard to social ties, 1227 (15.4 \%) participants would be considered marginallytied to family, and 1476 (18.5\%) would be considered marginally-tied with friends. Overall, 1225 (15.5 \%) scored as socially-isolated. On average, participants reported experiencing $1.6 \pm 1.4$ stressful life events in the past year, and 940 (12.3 \%) reported they had experienced stressful life events other than those listed. Finally, 1749 (21.2 \%) participants reported seeing any health care provider for mental health concerns, and where they did seek care it was most often received by a family medical doctor $(n=1027 ; 58.7 \%)$, counsellor $(n=256$, $14.6 \%)$ or psychologist ( $n=176 ; 10.1 \%)$.

Ethnocultural comparisons across all participants and the matched samples can be found in Table 3 for depressive symptoms, anxiety, social support, stressful life events and mental health service usage. As shown, Black participants reported significantly more stressful life events and less mental health service use than White participants, in both the overall and matched samples. South Asian participants reported significantly greater

Table 3 Comparison of psychosocial indicators between White and ethnocultural minority participants, in the overall and matched samples

\begin{tabular}{|c|c|c|c|c|c|c|}
\hline \multirow[t]{2}{*}{ Outcome Variable } & \multicolumn{3}{|l|}{ All Data } & \multicolumn{3}{|l|}{ Matched Data } \\
\hline & White $n=5120$ & Black $n=104$ & $p$ & White $n=2083$ & Black $n=100$ & $p$ \\
\hline Depressive symptoms & $16.38(6.26)$ & $16.25(6.60)$ & .668 & $16.51(5.95)$ & $16.11(6.63)$ & .370 \\
\hline Anxiety & $2.30(3.46)$ & $2.69(4.16)$ & .829 & $2.19(3.25)$ & $2.80(4.47)$ & .726 \\
\hline Life Events & $1.53(1.36)$ & $1.88(1.52)$ & .032 & $1.55(1.37)$ & $1.91(1.53)$ & .041 \\
\hline Social Support & $17.59(5.40)$ & $17.81(5.92)$ & .708 & $17.79(5.40)$ & $18.01(5.87)$ & .709 \\
\hline \multirow[t]{2}{*}{ Mental Health Services Use } & $.63(1.00)$ & $.33(.60)$ & $<.001$ & $.64(1.00)$ & $.33(.60)$ & $<.001$ \\
\hline & White $n=5120$ & South Asian $n=113$ & $p$ & White $n=2115$ & South Asian $n=111$ & $p$ \\
\hline Depressive symptoms & $16.38(6.26)$ & $16.31(6.25)$ & .870 & $16.06(5.88)$ & $16.22(6.22)$ & .889 \\
\hline Anxiety & $2.30(3.46)$ & $3.27(4.78)$ & .048 & 2.19 (3.19) & $3.27(4.82)$ & .048 \\
\hline Life Events & $1.53(1.36)$ & $1.65(1.44)$ & .302 & $1.43(1.29)$ & $1.67(1.44)$ & .088 \\
\hline Social Support & $17.59(5.40)$ & $17.41(6.11)$ & .756 & $17.55(5.25)$ & $17.45(6.14)$ & .862 \\
\hline \multirow[t]{2}{*}{ Mental Health Services Use } & $.63(1.00)$ & $.23(.57)$ & $<.001$ & $.57(.95)$ & $.23(.57)$ & $<.001$ \\
\hline & White $n=5120$ & East Asian $n=134$ & $p$ & White $n=2486$ & East Asian $n=132$ & $p$ \\
\hline Depressive symptoms & $16.38(6.26)$ & $16.37(5.70)$ & .792 & $16.11(5.82)$ & $16.36(5.74)$ & .533 \\
\hline Anxiety & $2.30(3.46)$ & $2.11(3.20)$ & .389 & $2.10(3.07)$ & $2.05(3.15)$ & .525 \\
\hline Life Events & $1.53(1.36)$ & $1.47(1.37)$ & .608 & $1.43(1.31)$ & $1.44(1.36)$ & .998 \\
\hline Social Support & $17.59(5.40)$ & $14.72(5.50)$ & $<.001$ & $17.44(5.40)$ & $14.73(5.53)$ & $<.001$ \\
\hline \multirow[t]{2}{*}{ Mental Health Services Use } & $.63(1.00)$ & $.20(.49)$ & $<.001$ & $.58(.97)$ & $.20(.49)$ & $<.001$ \\
\hline & White $n=5120$ & Southeast Asian $n=111$ & $p$ & White $n=1898$ & Southeast Asian $n=106$ & $p$ \\
\hline Depressive symptoms & $16.38(6.26)$ & $15.63(7.36)$ & .117 & $16.06(5.80)$ & $15.48(7.39)$ & .159 \\
\hline Anxiety & $2.30(3.46)$ & $2.64(4.16)$ & .829 & $2.11(3.06)$ & $2.57(4.20)$ & .808 \\
\hline Life Events & $1.53(1.36)$ & $1.42(1.50)$ & .211 & $1.44(1.33)$ & $1.37(1.46)$ & .347 \\
\hline Social Support & $17.59(5.40)$ & $16.90(5.93)$ & .229 & $17.21(5.46)$ & $16.82(5.99)$ & .512 \\
\hline \multirow[t]{2}{*}{ Mental Health Services Use } & $.63(1.00)$ & $.27(.57)$ & $<.001$ & $.55(.96)$ & $.28(.58)$ & .001 \\
\hline & White $n=5120$ & Aboriginal $n=21$ & $p$ & White $n=518$ & Aboriginal $n=20$ & $p$ \\
\hline Depressive symptoms & $16.38(6.26)$ & $22.00(10.77)$ & .033 & $17.00(6.30)$ & $22.40(10.89)$ & .055 \\
\hline Anxiety & $2.30(3.46)$ & $4.19(5.11)$ & .187 & $2.05(3.13)$ & $4.40(5.14)$ & .092 \\
\hline Life Events & $1.53(1.36)$ & $2.10(1.37)$ & .116 & $1.64(1.47)$ & $2.20(1.32)$ & .067 \\
\hline Social Support & $17.59(5.40)$ & $15.76(4.90)$ & .104 & $18.01(5.38)$ & $15.65(5.01)$ & .052 \\
\hline Mental Health Services Use & $.63(1.00)$ & $.95(1.07)$ & .072 & $.75(1.02)$ & $.90(1.07)$ & .367 \\
\hline
\end{tabular}


Table 4 Comparison of specific stressful life events, by ethnocultural background, in overall and matched samples

\begin{tabular}{|c|c|c|c|c|c|c|}
\hline \multirow[t]{2}{*}{ Stressful Life Event } & \multicolumn{3}{|l|}{ All Data } & \multicolumn{3}{|l|}{ Matched Data } \\
\hline & White $n=5120$ & Black $n=104$ & p & White $n=2083$ & Black $n=100$ & $p$ \\
\hline Illness/Injury & $539(10.5 \%)$ & $6(5.8 \%)$ & .143 & $228(10.9 \%)$ & $6(6.0 \%)$ & .137 \\
\hline Illness/Injury to rel/frnd & 2258 (44.1 \%) & 35 (33.7 \%) & .036 & $936(44.9 \%)$ & $35(35.0 \%)$ & .062 \\
\hline Death of a partner & 46 (.9 \%) & $3(2.9 \%)$ & .073 & 15 (.7 \%) & $3(3.0 \%)$ & .046 \\
\hline Death of a rel/frnd & 1505 (29.4 \%) & $44(42.3 \%)$ & .006 & $619(29.7 \%)$ & $43(43.0 \%)$ & .007 \\
\hline Divorce/Sep & $146(28.5 \%)$ & $10(9.6 \%)$ & .001 & 65 (3.1\%) & $10(10 \%)$ & .002 \\
\hline Finance diff & $968(18.9 \%)$ & $40(38.5 \%)$ & $<.001$ & $376(18.1 \%)$ & $38(38 \%)$ & $<.001$ \\
\hline Job Loss & $359(7.0 \%)$ & $11(10.6 \%)$ & .173 & 135 (6.5\%) & $10(10.0 \%)$ & .212 \\
\hline Retirement & 473 (9.2\%) & 9 (8.7 \%) & .999 & 175 (8.4 \%) & $8(8.0 \%)$ & .999 \\
\hline Crop Loss & $30(.6 \%)$ & $1(1.0 \%)$ & .465 & $15(.7 \%)$ & $1(1.0 \%)$ & .529 \\
\hline Business Failure & $72(1.4 \%)$ & $1(1.0 \%)$ & .999 & $37(1.8 \%)$ & $1(1.0 \%)$ & .999 \\
\hline \multirow[t]{2}{*}{ Family Conflict } & $869(17.0 \%)$ & $21(20.2 \%)$ & .359 & $369(17.7 \%)$ & $21(21.0 \%)$ & .422 \\
\hline & White $n=5120$ & South Asian $n=113$ & $p$ & White $n=2115$ & South Asian $n=111$ & $p$ \\
\hline Illness/Injury & $539(10.5 \%)$ & $12(10.6 \%)$ & .999 & $212(10.0 \%)$ & $12(10.8 \%)$ & .747 \\
\hline IIIness/Injury to rel/frnd & 2258 (44.1\%) & $55(48.7 \%)$ & .340 & $890(42.1 \%)$ & $55(49.5 \%)$ & .139 \\
\hline Death of a partner & 46 (.9 \%) & $1(.9 \%)$ & .999 & $13(.6 \%)$ & $1(.9 \%)$ & .512 \\
\hline Death of a rel/frnd & 1505 (29.4 \%) & 36 (31.9 \%) & .602 & $590(27.9 \%)$ & 35 (31.5) & .448 \\
\hline Divorce/Sep & $146(28.5 \%)$ & $2(1.8 \%)$ & .772 & $49(2.3 \%)$ & $2(1.8 \%)$ & .999 \\
\hline Finance diff & $968(18.9 \%)$ & $24(21.2 \%)$ & .544 & $356(16.8 \%)$ & $23(20.7 \%)$ & .300 \\
\hline Job Loss & $359(7.0 \%)$ & $11(9.7 \%)$ & .263 & $171(8.1 \%)$ & 11 (9.9\%) & .476 \\
\hline Retirement & 473 (9.2\%) & $8(7.1 \%)$ & .513 & $203(9.6 \%)$ & $8(7.2 \%)$ & .506 \\
\hline Crop Loss & $30(.6 \%)$ & $1(.9 \%)$ & .493 & $15(.7 \%)$ & $1(.9 \%)$ & .560 \\
\hline Business Failure & $72(1.4 \%)$ & $10(8.8 \%)$ & $<.001$ & $24(1.1 \%)$ & 10 (9.0\%) & $<.001$ \\
\hline \multirow[t]{2}{*}{ Family Conflict } & $869(17.0 \%)$ & $14(12.4 \%)$ & .252 & $319(15.1 \%)$ & $14(12.6 \%)$ & .585 \\
\hline & White $n=5120$ & East Asian $n=134$ & $p$ & White $n=2486$ & East Asian $n=132$ & $p$ \\
\hline Illness/Injury & $539(10.5 \%)$ & $11(8.2 \%)$ & .475 & 245 (9.9\%) & $11(8.3 \%)$ & .654 \\
\hline Illness/Injury to rel/frnd & $2258(44.1 \%)$ & $57(42.5 \%)$ & .792 & 1056 (42.5 \%) & 55 (41.7\%) & .928 \\
\hline Death of a partner & 46 (.9 \%) & $2(1.5 \%)$ & .348 & 17 (.7 \%) & $2(1.5 \%)$ & .248 \\
\hline Death of a rel/frnd & 1505 (29.4 \%) & $47(35.1 \%)$ & .179 & $707(28.4 \%)$ & $45(34.1 \%)$ & .168 \\
\hline Divorce/Sep & $146(28.5 \%)$ & $2(2.9 \%)$ & .591 & $56(2.3 \%)$ & $2(1.5 \%)$ & .999 \\
\hline Finance diff & 968 (18.9\%) & $20(14.9 \%)$ & .265 & $412(16.6 \%)$ & $18(13.6 \%)$ & .469 \\
\hline Job Loss & $359(7.0 \%)$ & $10(7.5 \%)$ & .863 & $164(6.6 \%)$ & $10(7.6 \%)$ & .593 \\
\hline Retirement & $473(9.2 \%)$ & $11(8.2 \%)$ & .879 & $220(8.8 \%)$ & $11(8.3 \%)$ & .999 \\
\hline Crop Loss & $30(.6 \%)$ & $1(.7 \%)$ & .552 & $15(.6 \%)$ & $1(.8 \%)$ & .564 \\
\hline Business Failure & $72(1.4 \%)$ & $5(3.7 \%)$ & .046 & $42(1.7 \%)$ & $5(3.8 \%)$ & .085 \\
\hline \multirow[t]{2}{*}{ Family Conflict } & $869(17.0 \%)$ & $17(12.7 \%)$ & .242 & $378(15.2 \%)$ & $16(12.1 \%)$ & .383 \\
\hline & White $n=5120$ & Southeast Asian $n=111$ & $p$ & White $n=1898$ & Southeast Asian $n=106$ & $p$ \\
\hline IIIness/Injury & $539(10.5 \%)$ & $12(10.8 \%)$ & .876 & $184(.097)$ & $12(11.3 \%)$ & .613 \\
\hline IIIness/Injury to rel/frnd & $2258(44.1 \%)$ & 35 (31.5 \%) & .009 & $808(42.6 \%)$ & $33(31.1 \%)$ & .020 \\
\hline Death of a partner & $46(.9 \%)$ & $0(0 \%)$ & .627 & $9(.5 \%)$ & $0(0 \%)$ & .999 \\
\hline Death of a rel/frnd & 1505 (29.4 \%) & 31 (27.9\%) & .833 & $533(28.1 \%)$ & $28(26.4 \%)$ & .824 \\
\hline Divorce/Sep & $146(28.5 \%)$ & $2(1.8 \%)$ & .771 & $50(2.6 \%)$ & $2(1.9 \%)$ & .999 \\
\hline Finance diff & $968(18.9 \%)$ & $30(27.0 \%)$ & .037 & $315(16.6 \%)$ & $27(25.5 \%)$ & .024 \\
\hline Job Loss & 359 (7.0\%) & $14(12.6 \%)$ & .037 & 137 (7.2 \%) & $12(11.3 \%)$ & .126 \\
\hline
\end{tabular}


Table 4 Comparison of specific stressful life events, by ethnocultural background, in overall and matched samples (Continued)

\begin{tabular}{|c|c|c|c|c|c|c|}
\hline Retirement & $473(9.2 \%)$ & $5(4.5 \%)$ & .095 & $180(9.5 \%)$ & $5(4.7 \%)$ & .119 \\
\hline Crop Loss & $30(.6 \%)$ & $1(.9 \%)$ & .487 & $16(.8 \%)$ & $0(0 \%)$ & .999 \\
\hline Business Failure & $72(1.4 \%)$ & $2(1.8 \%)$ & .671 & $33(1.7 \%)$ & $2(1.9 \%)$ & .708 \\
\hline \multirow[t]{2}{*}{ Family Conflict } & $869(17.0 \%)$ & 11 (9.9\%) & .053 & $289(15.2 \%)$ & $10(9.4 \%)$ & .123 \\
\hline & White $n=5120$ & Aboriginal $n=21$ & $p$ & White $n=518$ & Aboriginal $n=20$ & $p$ \\
\hline Illness/Injury & $539(10.5 \%)$ & $3(14.3 \%)$ & .480 & $60(11.6 \%)$ & $3(15 \%)$ & .719 \\
\hline Illness/Injury to rel/frnd & 2258 (44.1\%) & $12(57.1 \%)$ & .273 & $246(47.5 \%)$ & $12(60.0 \%)$ & .362 \\
\hline Death of a partner & $46(.9 \%)$ & $0(0 \%)$ & .999 & $6(1.2 \%)$ & $0(0 \%)$ & .999 \\
\hline Death of a rel/frnd & 1505 (29.4 \%) & $10(47.6 \%)$ & .090 & $163(31.5 \%)$ & $10(50.0 \%)$ & .091 \\
\hline Divorce/Sep & $146(28.5 \%)$ & $0(0 \%)$ & .999 & $16(3.1 \%)$ & $0(0 \%)$ & .999 \\
\hline Finance diff & $968(18.9 \%)$ & $4(19.0 \%)$ & .999 & $102(19.7 \%)$ & $4(20.0 \%)$ & .999 \\
\hline Job Loss & $359(7.0 \%)$ & $0(0 \%)$ & .396 & $36(6.9 \%)$ & $0(0 \%)$ & .387 \\
\hline Retirement & $473(9.2 \%)$ & $4(19.0 \%)$ & .124 & 35 (6.8 \%) & 4 (20.0 \%) & .049 \\
\hline Crop Loss & $30(.6 \%)$ & $0(0 \%)$ & .999 & $4(.8 \%)$ & $0(0 \%)$ & .999 \\
\hline Business Failure & $72(1.4 \%)$ & $0(0 \%)$ & .999 & 10 (1.9\%) & $0(0 \%)$ & .999 \\
\hline Family Conflict & 869 (17.0 \%) & 7 (33.3 \%) & .072 & 103 (19.9\%) & 7 (35.0 \%) & .151 \\
\hline
\end{tabular}

rel: a close relative; frnd: friend; Sep: separation; diff: difficulties

anxiety and less mental health service use than White participants in both the overall and matched samples. East Asian participants reported significantly lower social support and mental health service use than White participants in both the overall and matched samples. Southeast Asian participants reported significantly less mental health service use than White participants in both the overall and matched samples. Finally, in the overall sample, Aboriginal participants reported significantly greater depressive symptoms, and a trend toward greater mental health service use than White participants. In the matched samples, there were trends towards greater depressive symptoms, anxiety and stressful life events among Aboriginal participants when compared to White participants, and also a trend toward lower social support.

Since the matched White and South Asian participants differed significantly on the GAD-7, a test of measurement invariance was conducted using a multiple group structural equation model. More specifically, a multiple group confirmatory factor analysis was conducted with a single anxiety factor. Error covariances were included between chronic worrying and uncontrolled worrying, feeling afraid and uncontrolled worrying, and difficulty relaxing and restlessness. It was found that the factor loadings varied across the groups. Specifically, the factor loadings of the South Asian participants were significantly higher than those of the White participants on chronic worrying, difficulty relaxing, feeling afraid, feeling restless and uncontrolled worrying. There were no differences between the groups on being easily annoyed or nervous. All standardized factor loadings for both groups were greater than .5. The final model fit the data very well $\left(\chi^{2}[24 \mathrm{df}]=177.01, p<.001, \mathrm{RMSEA}=.035\right.$, CFI $=.992)$. Therefore the greater anxiety reported by the South Asian participants should not be overinterpreted given the stronger relationships between many of the items within the South Asian participants (relative to the White participants).

Since the matched White and East Asian participants differed significantly on the social support scale, a test of measurement invariance was again conducted using a multiple group structural equation model. More specifically, a multiple group confirmatory factor analysis was conducted with separate factors for support from friends and family. Error covariances were included between the associated relative and friend items "number seen or heard from" and "number can talk to about private matters". All factor loadings and the covariance between friend and family support were invariant across groups. Further, all standardized loadings for both groups were greater than .65. The final model had a good fit to the data $\left(\chi^{2}[12 \mathrm{df}]=230.05\right.$, $p<.001$, RMSEA $=.043$, CFI $=.986)$.

Ethnocultural comparisons in specific stressful life events can be found in Table 4. In the matched samples, Black participants were significantly more likely to report experiencing the death of a partner, death or a relative or friend, divorce or separation, and financial difficulties in the last year than White participants. South Asians were significantly more likely to report business failure than White participants. Aboriginal participants were significantly more 
likely to report retirement than White participants in the matched sample, with a trend toward more death among family and friends in the matched sample, and family conflict in the overall sample.
Ethnocultural comparisons in types of mental healthcare provider accessed can be found in Table 5. This was considered in all the most common ethnocultural groups as significant differences were observed in use overall,

Table 5 Comparison of specific mental healthcare provider accessed, by ethnocultural background, in overall and matched samples

\begin{tabular}{|c|c|c|c|c|c|c|}
\hline \multirow[t]{2}{*}{ Mental Health Care Provider Accessed } & \multicolumn{3}{|l|}{ All Data } & \multicolumn{3}{|l|}{ Matched Data } \\
\hline & White $n=5120$ & Black $n=104$ & $p$ & White $n=2083$ & Black $n=100$ & $p$ \\
\hline Psychiatrist & $535(10.4 \%)$ & $3(2.9 \%)$ & .008 & $224(10.8 \%)$ & $3(3.0 \%)$ & .011 \\
\hline Psychologist & $409(8.0 \%)$ & $3(2.9 \%)$ & .063 & $158(7.6 \%)$ & $3(3.0 \%)$ & .114 \\
\hline Psychotherapist & $169(3.3 \%)$ & $2(1.9 \%)$ & .777 & $72(3.5 \%)$ & $2(2.0 \%)$ & .774 \\
\hline Social Worker & 200 (3.9\%) & $1(1.0 \%)$ & .190 & $85(4.1 \%)$ & $1(1.0 \%)$ & .182 \\
\hline Counsellor & $553(10.8 \%)$ & $6(5.8 \%)$ & .110 & $235(11.2 \%)$ & $6(6.0 \%)$ & .139 \\
\hline Nurse & $34(0.7 \%)$ & $0(0 \%)$ & .999 & $14(.7 \%)$ & $0(0 \%)$ & .999 \\
\hline \multirow[t]{2}{*}{ Family Doctor } & 1324 (25.9 \%) & 19 (18.3\%) & .089 & $540(25.9 \%)$ & $18(18.0 \%)$ & .079 \\
\hline & White $n=5120$ & South Asian $n=113$ & $p$ & White $n=2115$ & South Asian $n=111$ & $p$ \\
\hline Psychiatrist & 535 (10.4 \%) & $3(2.7 \%)$ & .004 & $203(9.6 \%)$ & $3(2.7 \%)$ & .011 \\
\hline Psychologist & 409 (8.0\%) & $5(4.4 \%)$ & .215 & $160(7.6 \%)$ & $4(3.6 \%)$ & .137 \\
\hline Psychotherapist & 169 (3.3\%) & $1(.9 \%)$ & .273 & $51(2.4 \%)$ & $1(.9 \%)$ & .516 \\
\hline Social Worker & 200 (3.9\%) & $1(.9 \%)$ & .132 & $74(3.5 \%)$ & $1(.9 \%)$ & .179 \\
\hline Counsellor & $553(10.8 \%)$ & $5(4.4 \%)$ & .029 & 207 (9.8 \%) & 5 (4.5\%) & .068 \\
\hline Nurse & $34(0.7 \%)$ & $0(0 \%)$ & .999 & $10(.5 \%)$ & $0(0 \%)$ & .999 \\
\hline \multirow[t]{2}{*}{ Family Doctor } & 1324 (25.9 \%) & $11(9.7 \%)$ & $<.001$ & $510(24.1 \%)$ & $11(9.9 \%)$ & $<.001$ \\
\hline & White $n=5120$ & East Asian $n=134$ & $p$ & White $n=2486$ & East Asian $n=132$ & $p$ \\
\hline Psychiatrist & 535 (10.4 \%) & $5(3.7 \%)$ & .009 & $256(10.3 \%)$ & $4(3.0 \%)$ & .004 \\
\hline Psychologist & 409 (8.0 \%) & $8(6.0 \%)$ & .516 & $185(7.4 \%)$ & 8 (6.1\%) & .732 \\
\hline Psychotherapist & 169 (3.3\%) & $3(2.2 \%)$ & .803 & $69(2.8 \%)$ & $3(2.3 \%)$ & .999 \\
\hline Social Worker & $200(3.9 \%)$ & $2(1.5 \%)$ & .248 & $89(3.6 \%)$ & $2(1.5 \%)$ & .324 \\
\hline Counsellor & $553(10.8 \%)$ & $1(.7 \%)$ & $<.001$ & $248(10.0 \%)$ & $1(.8 \%)$ & $<.001$ \\
\hline Nurse & $34(0.7 \%)$ & $0(0 \%)$ & .999 & $14(.6 \%)$ & $0(0 \%)$ & .999 \\
\hline \multirow[t]{2}{*}{ Family Doctor } & 1324 (25.9 \%) & $8(6.0 \%)$ & $<.001$ & $569(22.9 \%)$ & $8(6.1 \%)$ & $<.001$ \\
\hline & White $n=5120$ & Southeast Asian $n=111$ & $p$ & White $n=1898$ & Southeast Asian $n=106$ & $p$ \\
\hline Psychiatrist & $535(10.4 \%)$ & $4(3.6 \%)$ & .017 & $171(9.0 \%)$ & $4(3.8 \%)$ & .074 \\
\hline Psychologist & 409 (8.0 \%) & $3(2.7 \%)$ & .047 & $142(7.5 \%)$ & $3(2.8 \%)$ & .081 \\
\hline Psychotherapist & 169 (3.3\%) & $0(0 \%)$ & .052 & $46(2.4 \%)$ & $0(0 \%)$ & .172 \\
\hline Social Worker & 200 (3.9\%) & 1 (.9\%) & .131 & $65(3.4 \%)$ & $.9 \%)$ & .258 \\
\hline Counsellor & $553(10.8 \%)$ & $2(1.8 \%)$ & .001 & $182(9.6 \%)$ & $2(1.9 \%)$ & .005 \\
\hline Nurse & $34(0.7 \%)$ & $0(0 \%)$ & .999 & $10(.5 \%)$ & $0(0 \%)$ & .999 \\
\hline \multirow[t]{2}{*}{ Family Doctor } & 1324 (25.9 \%) & 20 (18.0\%) & .062 & $425(22.4 \%)$ & $20(18.9 \%)$ & .471 \\
\hline & White $n=5120$ & Aboriginal $n=21$ & $p$ & White $n=518$ & Aboriginal $n=20$ & $p$ \\
\hline Psychiatrist & $535(10.4 \%)$ & $3(14.3 \%)$ & .477 & $62(12.0 \%)$ & $2(10.0 \%)$ & .999 \\
\hline Psychologist & 409 (8.0\%) & $5(23.8 \%)$ & .023 & 42 (8.1\%) & $5(25.0 \%)$ & .023 \\
\hline Psychotherapist & 169 (3.3 \%) & $0(0 \%)$ & .999 & $22(4.2 \%)$ & $0(0 \%)$ & .999 \\
\hline Social Worker & 200 (3.9\%) & $0(0 \%)$ & .999 & 19 (3.7\%) & $0(0 \%)$ & .999 \\
\hline Counsellor & $553(10.8 \%)$ & $3(14.3 \%)$ & .490 & 75 (14.5\%) & $3(15.0 \%)$ & .999 \\
\hline Nurse & $34(0.7 \%)$ & $0(0 \%)$ & .999 & 516 (0.4 \%) & $0(0 \%)$ & .999 \\
\hline Family Doctor & 1324 (25.9\%) & 9 (42.9 \%) & .084 & 169 (32.6 \%) & $8(40.0 \%)$ & .477 \\
\hline
\end{tabular}


and also among Aboriginal participants where a trend was observed. Black participants were significantly less likely to see a psychiatrist than their White counterparts. South Asian and East Asian participants were significantly less likely to see a psychiatrist or a family doctor than their White counterparts. Southeast Asian participants were significantly less likely to see a counsellor than their White counterparts. Finally, Aboriginal participants were significantly more likely to see a psychologist than White participants.

\section{Discussion}

The OHS serves as a rich source of information on the mental health status of ethnocultural minorities in Ontario. The findings likely reflect the experience of first and second generation immigrants to Ontario (e.g., South and Southeast Asian), but also reflect the experiences of long-established residents (e.g., Black, Aboriginal) who face a documented social disadvantage. Results showed that the most common ethnocultural minorities suffer a greater burden of psychosocial distress than their White counterparts, and, with the exception of Aboriginal Ontarians, were less likely to access care to treat this distress.

The literature regarding Canadian mental health more broadly illustrates that despite national diversity, few comprehensive studies have focused on mental health risk in relation to ethnicity. In addition, the overwhelming majority of such studies have focused specifically on depressive symptoms [12]. Previous studies have reported complex and variable associations when adults of specific national origins have been compared [30-37]. For example, higher rates of depressive symptoms have been reported in specific ethnic and sex-defined populations, like Chinese, Vietnamese, Portuguese, and Latin American women, compared to national figures [38]. In a study of Canadians of Ethiopian origin, Fenta et al. found rates that were lower or comparable to the national figures [39]. A number of studies have reported a lower prevalence of depressive symptoms in immigrants than those who are Canadian-born [12], in accordance with the so-called "healthy immigrant effect". Taken together, these data suggest that while ethnicity itself may not be predictive of depressive and anxiety rates, psychosocial factors associated with acculturation and social disadvantage may contribute to the increased mental health risk seen in ethnic minority populations.

In this study, ethnocultural minorities reported lower access to mental health care providers. Given that mental health risk has been illustrated to be higher in these groups, minority Ontarians, even under universal health care, are likely suffering psychological distress without accessing effective treatments. Previous research has similarly shown that ethnic minorities make less use of services than do the White majority, although there is limited understanding as to why this is the case [40]. Factors such as inability to effectively communicate and navigate within Canada's health care system may play a role. Insufficient access could also be linked to tendencies in some ethnocultural groups to seek healthcare that addresses physical illness but not for mental health, screening or preventive care services. There may also be ethnocultural differences associated with the propensity to seek support from religious sources, in contrast to secular sources, for mental health problems [41].

Caution is warranted in interpreting the findings. First, the generalizability is limited by the low response rate. The generalizability of the sample to the population of Ontario more broadly remains unknown. Moreover, given that the surveys were administered in English, generalizability is limited to immigrants with Englishlanguage proficiency. Second, no structured diagnostic interviews were performed, and while psychometricallyvalidated measures of depressive symptoms and anxiety in particular were administered, it is unknown how many participants would be diagnosed with a true mental disorder. Third, the lack of data on place of birth and generation status, immigration/refugee status, years in Canada, and English-language proficiency in the $\mathrm{OHS}$ dataset limits the potential to interpret these findings. Finally, multiple comparisons were performed, which may have inflated type I error.

\section{Conclusion}

Mental health disparities are observed in Ontarians, with the most common or prevalent ethnocultural minorities suffering a higher level of psychosocial distress than their White counterparts. Furthermore, with the exception of Aboriginal Ontarians, these ethnocultural minorities are also less likely to access treatments that address distress. In the context of few Canadian studies on mental health risk in relation to ethnicity, our study indicates that the social determinants of health may be important factors to consider in future.

\section{Abbreviations \\ CES-D: Center for Epidemiological Studies Depressive Symptoms Scale; GAD- 7: Generalized Anxiety Disorder Scale; OHS: Ontario Health Study.}

\section{Competing interests}

All authors declare that they have no competing interests.

\section{Authors' contributions}

SLG contributed to the concept, analysis, and interpretation; drafted the manuscript. YT contributed to the analysis, and interpretation; drafted the manuscript. RC contributed to the data analysis and interpretation; drafted the manuscript. HN contributed to the data acquisition, analysis, and interpretation; drafted the manuscript. PR contributed to the data interpretation; critically revised the manuscript. J contributed to the concept and design, critically revised the manuscript. All authors read and approved the final manuscript. 


\section{Acknowledgements}

Data used for this research were made available by the Ontario Health Study, which is funded by the Ontario Institute for Cancer Research, the Canadian Partnership Against Cancer, Cancer Care Ontario, and Public Health Ontario. We thank the participants in the Ontario Health Study.

\section{Author details \\ 'School of Kinesiology and Health Science, York University, Toronto, ON, Canada. ${ }^{2}$ Cardiac Rehabilitation and Prevention Program, Toronto Rehabilitation Institute, University Health Network, Toronto, ON, Canada. ${ }^{3}$ Department of Psychology, York University, Toronto, ON, Canada. ${ }^{4}$ Department of Medicine, University of Toronto, Toronto, ON, Canada. ${ }^{5}$ Bethune College 368 York University, 4700 Keele Street, Toronto, ON, Canada.}

Received: 5 February 2015 Accepted: 22 February 2016 Published online: 26 February 2016

\section{References}

1. Dewa CS, Chau N, Dermer S. Examining the comparative incidence and costs of physical and mental health-related disabilities in an employed population. J Occup Environ Med. 2010;52:758-62.

2. Smetanin P, Stiff D, Briante C, Adair C, Ahmad S, Khan M: The Life and Economic Impact of Major Mental IIInesses in Canada: 2011 to 2041. Toronto, Canada; 2011

3. Health Canada: A Report on Mental IIlnesses in Canada. Ottawa, Canada; 2002.

4. Lim K-L, Jacobs P, Ohinmaa A, Schopflocher D, Dewa CS. A new populationbased measure of the economic burden of mental illness in Canada. Chronic Dis Can. 2008:28:92-8.

5. Leray E, Camara A, Drapier D, Riou F, Bougeant N, Pelissolo A, Lloyd KR, Bellamy V, Roelandt JL, Millet B. Prevalence, characteristics and comorbidities of anxiety disorders in France: results from the "Mental Health in General Population" survey (MHGP). Eur Psychiatry. 2011;26:339-45.

6. Akhtar-Danesh N, Landeen J. Relation between depression and sociodemographic factors. Int J Ment Health Syst. 2007;1:4

7. Bovier PA, Chamot E, Perneger TV. Perceived stress, internal resources, and social support as determinants of mental health among young adults. Qual Life Res. 2004;13:161-70.

8. Henken HT, Huibers MJH, Churchill R, Restifo K, Roelofs J. Family therapy for depression. Cochrane database Syst Rev. 2007;18, CD006728.

9. Hofstede GH. Culture's Consequences: Comparing Values, Behaviors, Institutions and Organizations Across Nations. Secondth ed. Thousand Oaks, CA: SAGE Publications; 2001.

10. Immigration and Ethnocultural Diversity in Canada [http://www12.statcan. gc.ca/nhs-enm/2011/as-sa/99-010-x/99-010-x2011001-eng.pdf]

11. Projections of the diversity of the Canadian population [http://www.statcan. gc.ca/daily-quotidien/100309/dq100309a-eng.htm]

12. Hansson EK, Tuck A, Lurie S, McKenzie K. Rates of mental illness and suicidality in immigrant, refugee, ethnocultural, and racialized groups in Canada: a review of the literature. Can J Psychiatry. 2012;57:111-21.

13. Hansson, E., Tuck, A., Lurie, S. \& McKenzie, K., for the Task Group of the Services Systems Advisory Committee: Mental Health Commission of Canada. Improving mental health services for immigrant, refugee, ethnocultural and racialized groups: Issues and options for service improvement [report]. 2010. http://www.mentalhealthcommission.ca/English/system/files/ private/Diversity_Issues_Options_Report_ENG_0.pdf.

14. Kirmayer $L J$, Brass GM, Tait CL. The mental health of Aboriginal peoples: transformations of identity and community. Can J Psychiatry. 2000:45:607-16.

15. Beiser M. The health of immigrants and refugees in Canada. Can J public Heal Rev Can santé publique. 2005;96 Suppl 2:S30-44.

16. About the Study [https://www.ontariohealthstudy.ca/en/about-study]

17. Radloff LS. The CES-D scale: a self-report depression scale for research in the general population. Appl Psychol Meas. 1977;1:385-401.

18. Gupta R, Punetha D, Diwan S. The revised CES-D scale for caregivers of the elderly in India. Int J Aging Hum Dev. 2006;62:61-78.

19. Boey KW. Cross-validation of a short form of the CES-D in Chinese elderly. Int J Geriatr Psychiatry. 1999;14:608-17.

20. Irwin $\mathrm{M}$, Artin $\mathrm{KH}$, Oxman MN. Screening for depression in the older adult: criterion validity of the 10-item Center for Epidemiological Studies Depression Scale (CES-D). Arch Intern Med. 1999;159:1701-4.
21. Spitzer RL, Kroenke K, Williams JBW, Löwe B. A brief measure for assessing generalized anxiety disorder: the GAD-7. Arch Intern Med. 2006;166:1092-7.

22. Kroenke K, Spitzer RL, Williams JBW, Monahan PO, Löwe B. Anxiety disorders in primary care: prevalence, impairment, comorbidity, and detection. Ann Intern Med. 2007;146:317-25.

23. Lubben J, Blozik E, Gillmann G, lliffe S, von Renteln KW, Beck JC, Stuck AE. Performance of an abbreviated version of the Lubben Social Network Scale among three European community-dwelling older adult populations. Gerontologist. 2006;46:503-13.

24. Wright AF, Carothers AD, Campbell H. Gene-environment interactions-the BioBank UK study. Pharmacogenomics J. 2002:2:75-82.

25. Rosengren A, Hawken S, Ounpuu S, Sliwa K, Zubaid M, Almahmeed WA, Blackett KN, Sitthi-amorn C, Sato H, Yusuf S. Association of psychosocial risk factors with risk of acute myocardial infarction in 11119 cases and 13648 controls from 52 countries (the INTERHEART study): case-control study. Lancet. 2004;364:953-62.

26. Ho D, Imai K, King G, Stuart E. Matchlt: nonparametric preprocessing for parametric causal inference. J Stat Softw. 2011;42:1-28.

27. Hu L, Bentler PM. Cutoff criteria for fit indexes in covariance structure analysis: Conventional criteria versus new alternatives. Struct Equ Model A Multidiscip J. 1999;6:1-55.

28. R Core Team: R: A Language and Environment for Statistical Computing. 2014.

29. Geographic units by province and territory, 2011 Census [http://www. statcan.gc.ca/pub/92-195-x/2011001/other-autre/geograph/uni-eng.htm]

30. deGraaf R, ten Have M, man Gool C, Van Dorsselaer S. Prevalence of mental disorders and trends from 1996 to 2009. Results from the Netherlands mntal health survey and incidence study-2. Soc Psychiatry Psychiatr Epidemiol. 2012;47:203-13.

31. Gonzalez HM, Tarraf W, Whitfield KE, Vega WA. The epidemiology of depression and ethnicity in the United States. J Psychiatr Res. 2010;44:1043-51.

32. Gu L, Xie J, Long J, Chen Q, Chen Q, Pan R, Yan Y, Wu G, Liang B, Tan J, Xie W, Wei B, Su L. Epidemiology of major depressive disorder in mainlan china: A systematic review. PLoS One. 2013:13, e65356.

33. Gum AM, King-Kallimanis B, Kohn R. Prevalence of mood, anxiety, and substance-abuse disorders for older Americans in the national comorbidity survey - replication. Am J Geeriatr Psychiatry. 2009;17:769-81.

34. Liu J, Yan F, Ma X, Guo HL, Tang YL, Rakofsky JJ, Wu XM, Li XQ, Zhu H, Guo XB, Yang Y, Li P, Cao XD, Li HY, Li ZB, Wang P, Xu QY. Prevalence of major depressive disorder and socio-demographic correlates: Results of a representative household epidemiological survey in Beijing, China. J Affect Disord. 2015;179:74-81.

35. Sadeghirad B, Hadhdoost AA, Amin-Esmaeili M, Ananloo ES, Ghaeli P, Rahimi-Movaghar A, Talebian E, Pourkhandani A, Noorbala AA, Barooti E. Epidemiology of major depressive disorder in Iran: a systematic review and meta-analysis. Int J Prev Med. 2010;1:81-91.

36. Topuzoglu A, Binbay T, Ulas H, Elbi H, Tanik FA, Zagli N, Alptekin K. The epidemiology of major depressive disorder and subthreshold depression in Izmir, Turkey: Prevalence, socioeconomic differences, impairment and helpseeking. J Affect Disord. 2015;181:78-86.

37. Toselli S, Gualdi-Russo E, Marzouk D, Sundquist J, Sundquist K. Psychosocial health among immigrants in central and southern Europe. Eur J Public Health. 2014;24 Suppl 1:26-30.

38. Franks F, Faux SA. Depression, stress, mastery, and social resources in four ethnocultural women's groups. Res Nurs Health. 1990;13:283-92.

39. Fenta H, Hyman I, Noh S. Determinants of Depression Among Ethiopian Immigrants and Refugees in Toronto. J Nerv Ment Dis. 2004;192:363-72.

40. Morgan C, Mallett R, Hutchinson G, Leff J. Negative pathways to psychiatric care and ethnicity: the bridge between social science and psychiatry. Soc Sci Med. 2004:58:739-52

41. Ransford HE, Carrillo FR, Rivera Y. Health care-seeking among Latino immigrants: blocked access, use of traditional medicine, and the role of religion. J Health Care Poor Underserved. 2010;21:862-78. 